\title{
Qualification and performance of the Low-Energy X-ray Reflectometer (LEXR)
}

Henriksen, P. L.; Christensen, F. E.; Massahi, S.; Ferreira, D. D.M.; Svendsen, S.; Gellert, N.; Vu, L. M.; Jegers, A. S.; Shortt, B.

Published in:

Space Telescopes and Instrumentation 2020: Ultraviolet to Gamma Ray

Link to article, DOI:

$10.1117 / 12.2562242$

Publication date:

2020

Document Version

Publisher's PDF, also known as Version of record

Link back to DTU Orbit

Citation (APA):

Henriksen, P. L., Christensen, F. E., Massahi, S., Ferreira, D. D. M., Svendsen, S., Gellert, N., Vu, L. M., Jegers, A. S., \& Shortt, B. (2020). Qualification and performance of the Low-Energy X-ray Reflectometer (LEXR). In J-W. A. den Herder, S. Nikzad, \& K. Nakazawa (Eds.), Space Telescopes and Instrumentation 2020: Ultraviolet to Gamma Ray (Vol. 11444). [114444J] SPIE - International Society for Optical Engineering. Proceedings of SPIE The International Society for Optical Engineering Vol. 11444 https://doi.org/10.1117/12.2562242

\section{General rights}

Copyright and moral rights for the publications made accessible in the public portal are retained by the authors and/or other copyright owners and it is a condition of accessing publications that users recognise and abide by the legal requirements associated with these rights.

- Users may download and print one copy of any publication from the public portal for the purpose of private study or research.

- You may not further distribute the material or use it for any profit-making activity or commercial gain

- You may freely distribute the URL identifying the publication in the public portal 


\section{Qualification and performance of the Low-Energy X-ray Reflectometer (LEXR)}

Henriksen, P. L., Christensen, F. E. , Massahi, S., Ferreira, D. D. M., Svendsen, S., et al.

P. L. Henriksen, F. E. Christensen, S. Massahi, D. D. M. Ferreira, S.

Svendsen, N. Gellert, L. M. Vu, A. S. Jegers, B. Shortt, "Qualification and performance of the Low-Energy X-ray Reflectometer (LEXR)," Proc. SPIE 11444, Space Telescopes and Instrumentation 2020: Ultraviolet to Gamma Ray, 114444J (13 December 2020); doi: 10.1117/12.2562242

SPIE. Event: SPIE Astronomical Telescopes + Instrumentation, 2020, Online Only 


\title{
Qualification and performance of the Low-Energy X-ray Reflectometer (LEXR)
}

\author{
P. L. Henriksen ${ }^{a}$, F. E. Christensen ${ }^{a}$, S. Massahi ${ }^{a}$, D. D. M. Ferreira ${ }^{a}$, S. Svendsen ${ }^{a}$, N. \\ Gellert $^{a}$, L. M. Vu ${ }^{a}$, A. 'S Jegers ${ }^{a}$, B. Shortt ${ }^{b}$

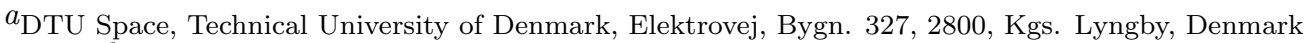

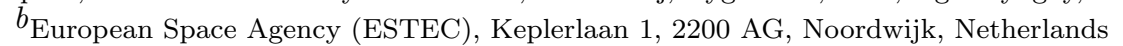

\begin{abstract}
A state-of-the-art Low-Energy X-ray Reflectometer (LEXR) is in operation at DTU Space with the main purpose of characterizing coatings for the Advanced Telescope for High-ENergy Astrophysics (ATHENA), a selected Lclass ESA mission. In particular, soft materials are difficult to characterize at higher energies so the $1.487 \mathrm{keV}$ beamline compliments our existing $8.048 \mathrm{keV}$ reflectometer, allowing for a more complete understanding of thinfilm X-ray characteristics.

Documenting and qualifying the as-deposited coatings for X-ray telescope optics is of crucial importance, both to ensure that the effective area requirements are met, but also to quantify any temporal evolution in coating characteristics as well as the impact of manufacturing process parameters on mirror performance. It is notably relevant in the case of ATHENA, as there is a desire to enhance the low-energy performance by using a low-Z material overcoating on top of the Ir coating.

We report on the commissioning and qualify the performance of the as-built $1.487 \mathrm{keV}$ reflectometer as well as discuss measurement repeatability and system limitations.
\end{abstract}

Keywords: LEXR, X-ray reflectometry, ATHENA, optical coatings, X-ray optics, thin-film characterization, grazing incidence, $\mathrm{X}$-ray telescopes

\section{INTRODUCTION}

A number of future spaceborne and ground-based observatories equipped with X-ray telescopes rely on high throughput of the optics at low energies. ${ }^{1-3}$ It is crucial to be able to investigate these optics at relevant energies to obtain a detailed understanding of the performance and behavior of their mirrors.

To investigate thin-film performance of especially low- $Z$ materials and the effects of hydrocarbons and other surface contaminants, as well as mirror manufacturing process steps, ${ }^{4-6}$ DTU Space has commissioned a stateof-the-art Low-Energy X-ray Reflectometer (LEXR), the design of which has been reported previously. ${ }^{7}$ LEXR primarily uses a microfocus $\mathrm{Al}$ source and a $\mathrm{W} / \mathrm{Si}$ multilayer monochromator to produce a $1.487 \mathrm{keV}$ beam but can be equipped with other monochromators and sources should a beamline at another energy be desired. Due to the low-pressure requirement of the X-ray source, the operation pressure of the system is below $9 \times 10^{-6} \mathrm{mbar}$ and the beam path is just under $2 \mathrm{~m}$, meaning that the photon attenuation factor for the

$1.487 \mathrm{keV}$ setup is of $\mathcal{O}\left(10^{-8}\right)$.

Qualification of a low-Z overcoat on a dense film gets increasingly difficult with higher energies due to their reduced reflectance but X-ray reflectivity measurements at low energies are more susceptible to anomalous effects due to e.g. hydrocarbons as well as attenuation caused by dust or other contaminants on the reflective surface. For this reason, it is important to understand the sources of uncertainty on the measurements in order to determine not only the capabilities of an X-ray reflectivity (XRR) system but also its limitations.

In this paper we present the qualification of LEXR. We examine the performance of each of the components and demonstrate how sample information can be extracted by performing different types of measurements. Finally, we discuss the uncertainty on measurements and limitations on information we can extract from the system.

Further author information - send correspondence to:

Peter Lindquist Henriksen, e-mail: plihen@space.dtu.dk

Space Telescopes and Instrumentation 2020: Ultraviolet to Gamma Ray, edited by Jan-Willem A. den Herder

Shouleh Nikzad, Kazuhiro Nakazawa, Proc. of SPIE Vol. 11444, 114444J · (c) 2020 SPIE

CCC code: $0277-786 \mathrm{X} / 20 / \$ 21 \cdot$ doi: $10.1117 / 12.2562242$

Proc. of SPIE Vol. 11444 114444J-1 


\section{DYNAMIC RANGE AND BEAM STABILITY}

The reflectometer is equipped with a 2D CCD detector, the noise level of which is highly dependent on temperature. The detector is actively cooled by a Peltier element that can be set to cool the sensor down to $-80{ }^{\circ} \mathrm{C}$. At high temperatures, the total noise is dominated by dark current and is thus proportional to exposure time. When cooled, the readout noise is dominant, effectively making the total noise almost independent of exposure time. Figure 1 shows measurements of the noise as function of temperature and exposure time at different readout frequencies and gain settings. From these measurements it is decided to use a standard temperature setting of $-50{ }^{\circ} \mathrm{C}$ as a trade-off between a noise level close to independence of exposure time while at the same time minimizing strain on the Peltier element. The noise obtained in a region of interest (ROI) is independent of any light source on neighboring pixels in the detector. In addition to the options shown, a readout frequency setting of $3 \mathrm{MHz}$ is used only for alignment purposes.
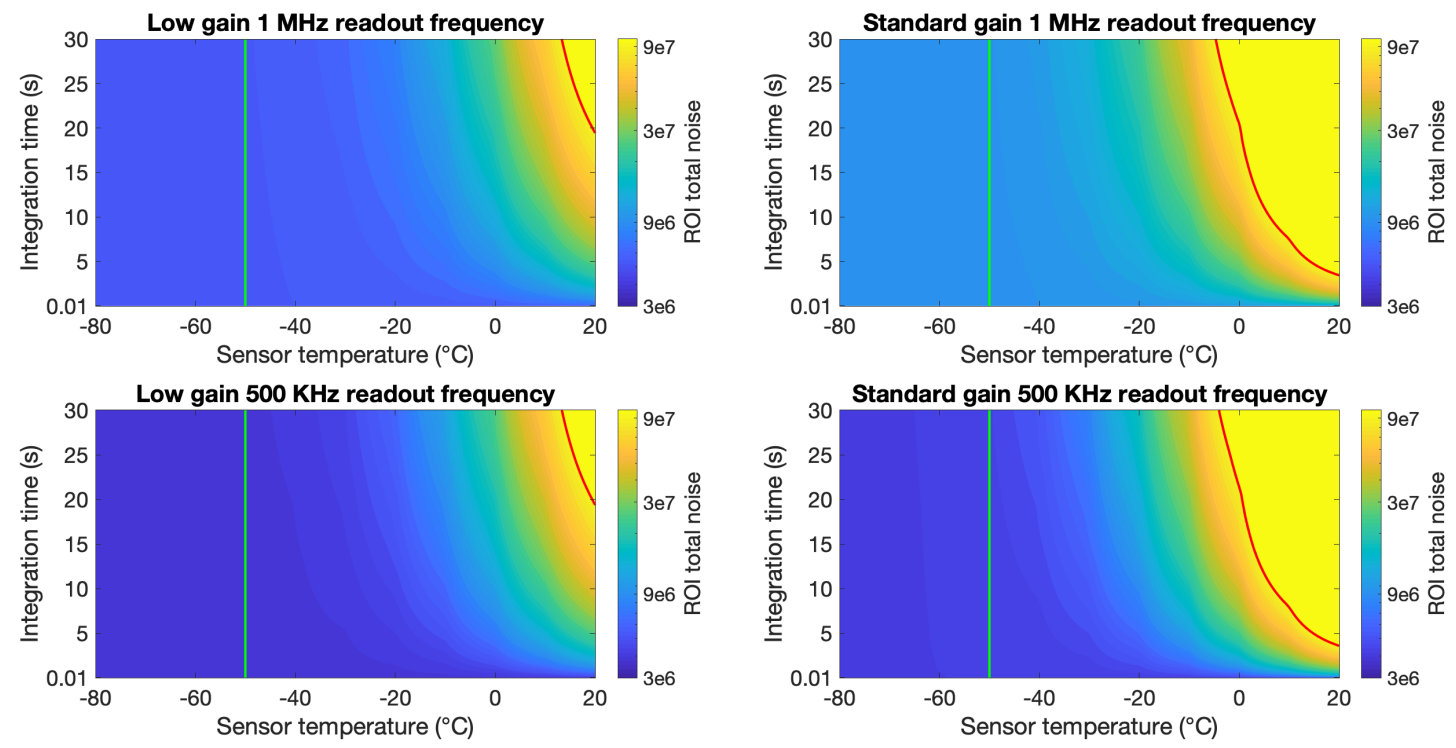

Figure 1: Total integrated noise in a suitable region of interest $(60 \times 110$ pixels $)$, each plot shows a natural cubic spline interpolation from $11 \times 12$ data points. The red line marks the point of detector saturation. The green line indicates the sensor temperature of $-50{ }^{\circ} \mathrm{C}$ used for standard measurements.

During specular measurements exposure times of up to $30 \mathrm{~s}$ at each data point are used. Figure 2 shows specular and non-specular scatter measurements of a NuSTAR-type ${ }^{8}$ mirror sample coated with $10 \mathrm{~nm} \mathrm{Ir}$, demonstrating the ability of LEXR to measure up to a grazing angle of $\theta=17.5 \mathrm{deg}$ and with a dynamic range of almost 8 orders of magnitude. To obtain such a high dynamic range, the detector was cooled to $-70{ }^{\circ} \mathrm{C}$, and exposure times of up $300 \mathrm{~s}$ were used for each data point, making such measurements rather time-consuming. If the reflectometer is used with a different source setup and operated in atmospheric conditions, the detector must be kept above the dew point to prevent condensation. 


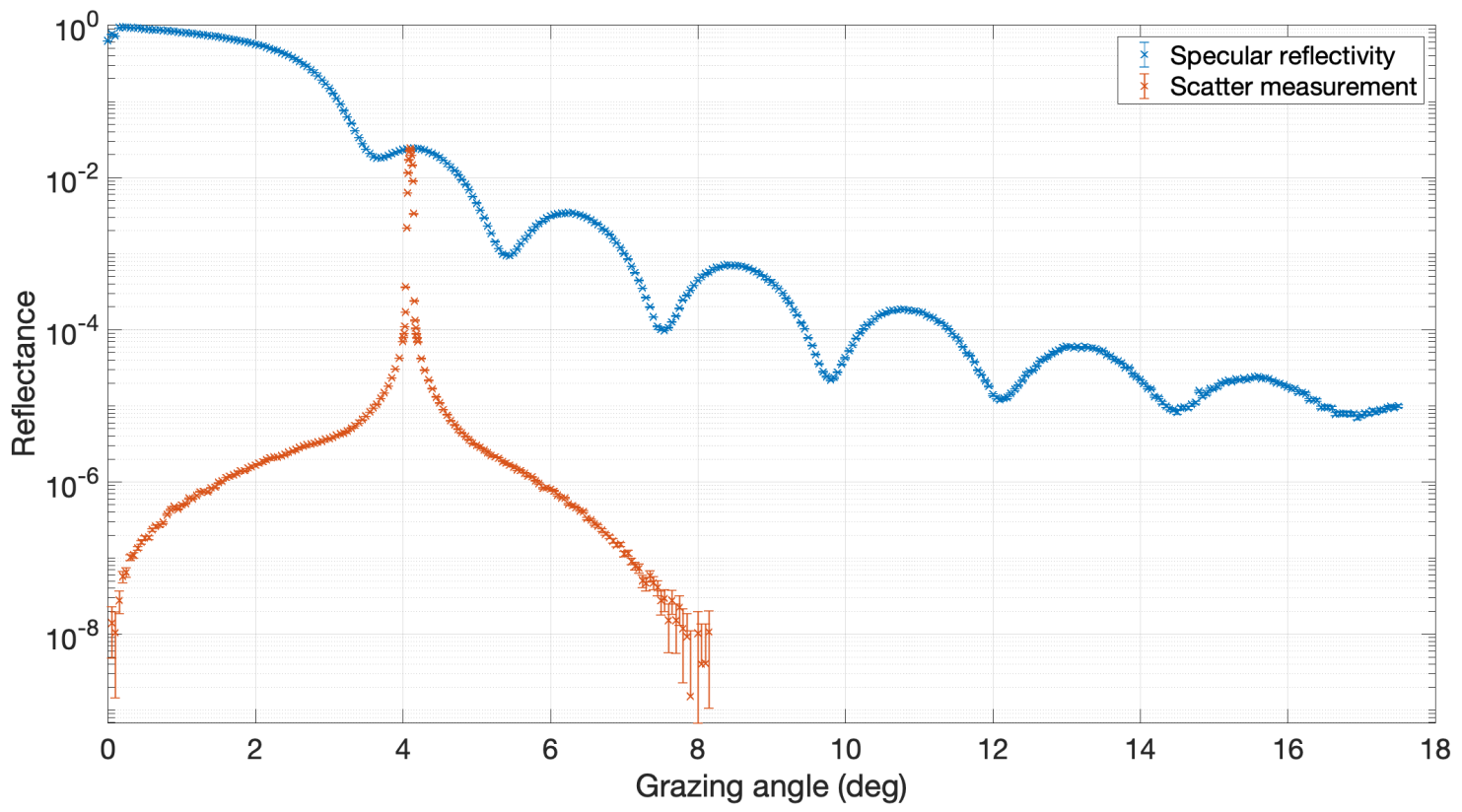

Figure 2: Specular and non-specular reflectivity measurements of a NuSTAR-type mirror substrate coated with $10 \mathrm{~nm}$ Ir.

The typical time to complete a specular reflectivity measurement with 6 orders of magnitude is around 2.5 hours. Figure 3 shows measurements of the pressure in the reflectometer chamber and the long-term beam intensity over the course of 14 hours. The relative standard deviation of measured intensity (signal + noise) over any 2 hour period varies between $0.42 \%$ and $0.56 \%$ with a mean around $0.47 \%$. At shallow angles where the reflected signal is strong, the variation is mainly caused by flux variation from the beam (described in Section 6.1). At large grazing angles the largest contributor to variation in the measured beam intensity is by far variation in the noise level of the detector. Exposure times here are typically $30 \mathrm{~s}$, and over such a period, the mean peak-to-valley is around $1.5 \%$ with a maximum of $2.7 \%$.
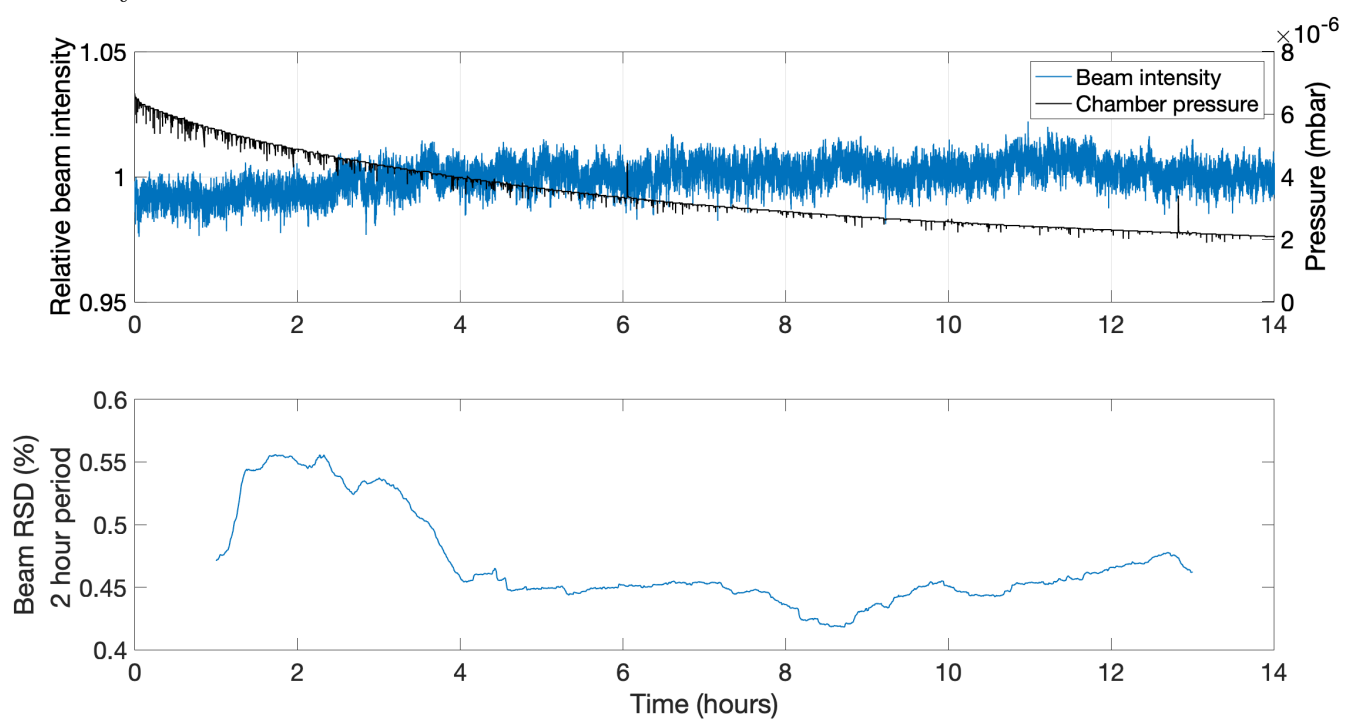

Figure 3: Top: Evolution of chamber pressure and relative beam intensity measured over a period of 14 hours with a sampling frequency of $1 \mathrm{~s}$. Bottom: Relative standard deviation (RSD) of recorded beam intensity over any 2 hour period within total measurement duration. 


\section{BEAM PURITY}

A W/Si multilayer coated mirror is used to monochromatize the beam for $1.487 \mathrm{keV}$ photons. The monochromator is designed for Bragg reflection of $\mathrm{Al} \mathrm{K}_{\alpha}$ photons at $6.54 \mathrm{deg}$. Figure 4 shows a reflectivity measurement of the monochromator around this angle performed using LEXR. The scan of the monochromator shows peak reflectance of $45 \%$ at $6.53 \mathrm{deg}$, and the best fit gives a W/Si d-spacing of $3.76 \mathrm{~nm}$ with $\Gamma=0.3$ for the 80 bilayers.

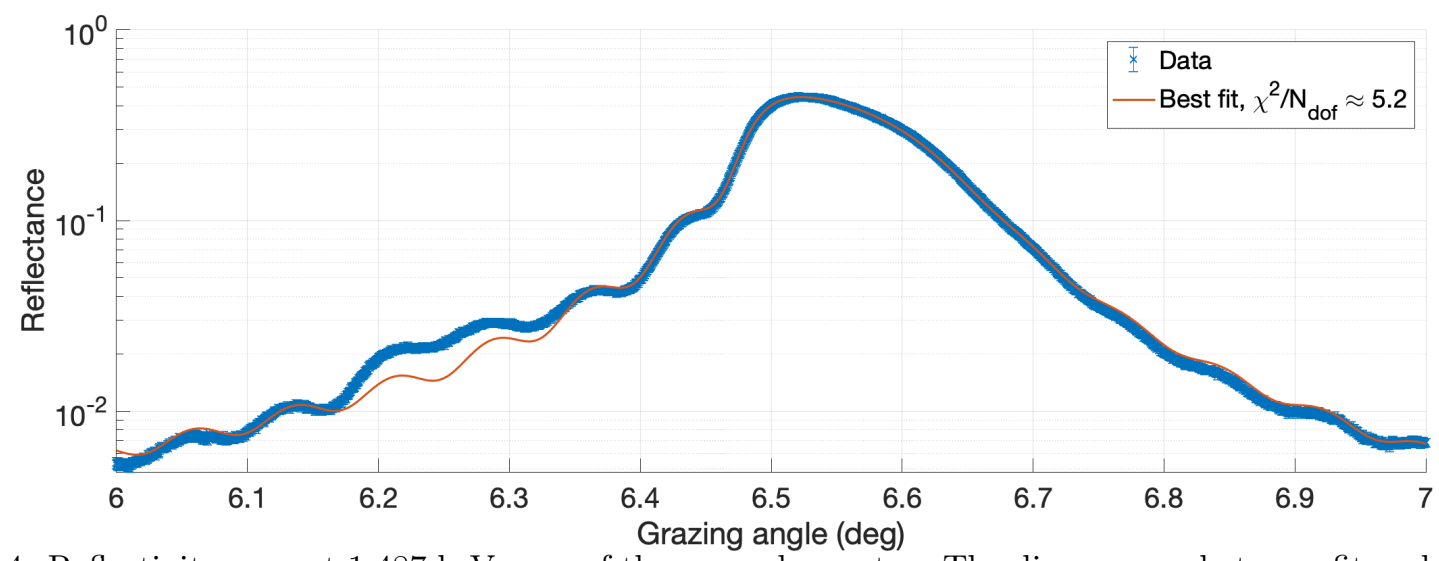

Figure 4: Reflectivity scan at $1.487 \mathrm{keV}$ scan of the monochromator. The discrepancy between fit and measured data at $6.2-6.3 \mathrm{deg}$ is due to the Bragg reflection of the $1.55 \mathrm{keV} \mathrm{Al} \mathrm{K}_{\beta}$ photons from this multilayer and is also the cause of the low $\chi^{2}$ probability even with large error bars.

The discrepancy in measured intensity in the region 6.2-6.3 deg corresponds to the location of the Bragg peak of the multilayer for the $1.55 \mathrm{keV} \mathrm{Al} \mathrm{K}_{\beta}$ edge. The measured signal here is $0.022 / 0.015 \approx 1.5$ times higher than expected for pure $\mathrm{Al} \mathrm{K}_{\alpha}$ photons. The measured peak reflectance at $6.54 \mathrm{deg}$ is slightly higher than expected from the model. This discrepancy matches exactly the ratio of $\mathrm{Al} \mathrm{K}_{\beta}$ to $\mathrm{Al} \mathrm{K}_{\alpha}$ photons convoluted with the $\mathrm{Al}$ $\mathrm{K}_{\beta}$ reflection at this angle. This Bragg peak has a reflectance of $43 \%$ of $\mathrm{Al} \mathrm{K}_{\beta}$ photons and $15 \%$ of $1.487 \mathrm{keV}$ photons, so the source flux of $1.55 \mathrm{keV}$ photons is around 0.174 that of $1.487 \mathrm{keV}$ photons. At the 6.54 degrees incidence angle of the monochromator, the reflectance of $1.487 \mathrm{keV}$ photons is $43.5 \%$ and that of the $1.55 \mathrm{keV}$ photons is $1.7 \%$. With the ratio of $\mathrm{K}_{\alpha}$ to $\mathrm{K}_{\beta}$ photons measured, this results in a beam purity of $99.3 \%$.

\subsection{Beam polarization}

The radiation from the X-ray source is assumed unpolarized so the polarization of the beam impinging on samples comes from the reflection off the Kirkpatrick-Baez (KB) mirrors in the source housing and the monochromator. The polarization by reflection is a function of the refractive index of the interface and the angle of incidence. The reflection angles off the KB mirrors are small $(2 \theta=2.1 \mathrm{deg}$ and $1.5 \mathrm{deg})$ and as the refractive index in the $\mathrm{X}$-ray regime is close to unity, the beam collimated by the KB mirrors is partially polarized only on the order of $0.1 \%$. The incidence angle on the monochromator is substantially higher at $\theta=6.54 \mathrm{deg}$ which, using the best-fit model to the monochromator scan, yields a partial s-polarization of $2.5 \%$. Polarization of the beam can not be distinguished by the detector but, albeit a small effect, sample reflectivity is dependent on beam polarization with increasing importance as function of incidence angle. The polarization in this case is so small that the effect on reflectance is less than one percent. 


\section{RESOLUTION AND REPEATABILITY}

\subsection{Instrument function}

Figure 5 shows a measurement of the scattering from the reflectometer components (collimating mirrors, slits, monochromator, etc.) themselves. The measurement has a FWHM of 0.14 deg but more importantly it shows that instrument scatter is $\mathcal{O}\left(10^{-4}\right)$ or less, meaning the scatter instrument function is negligible during measurements as it folds into the specular reflectivity measurements in the second order. At rocking curve measurements, the specular part of the instrument scatter will be reflected outside the detector when $\omega \gtrsim \pm 0.5$ deg from the specular direction. If sample scatter measurements were instead carried out at fixed incidence by scanning the detector angle, a deconvolution between instrument scatter and measured scatter would have to be performed to obtain the scattering from the sample.

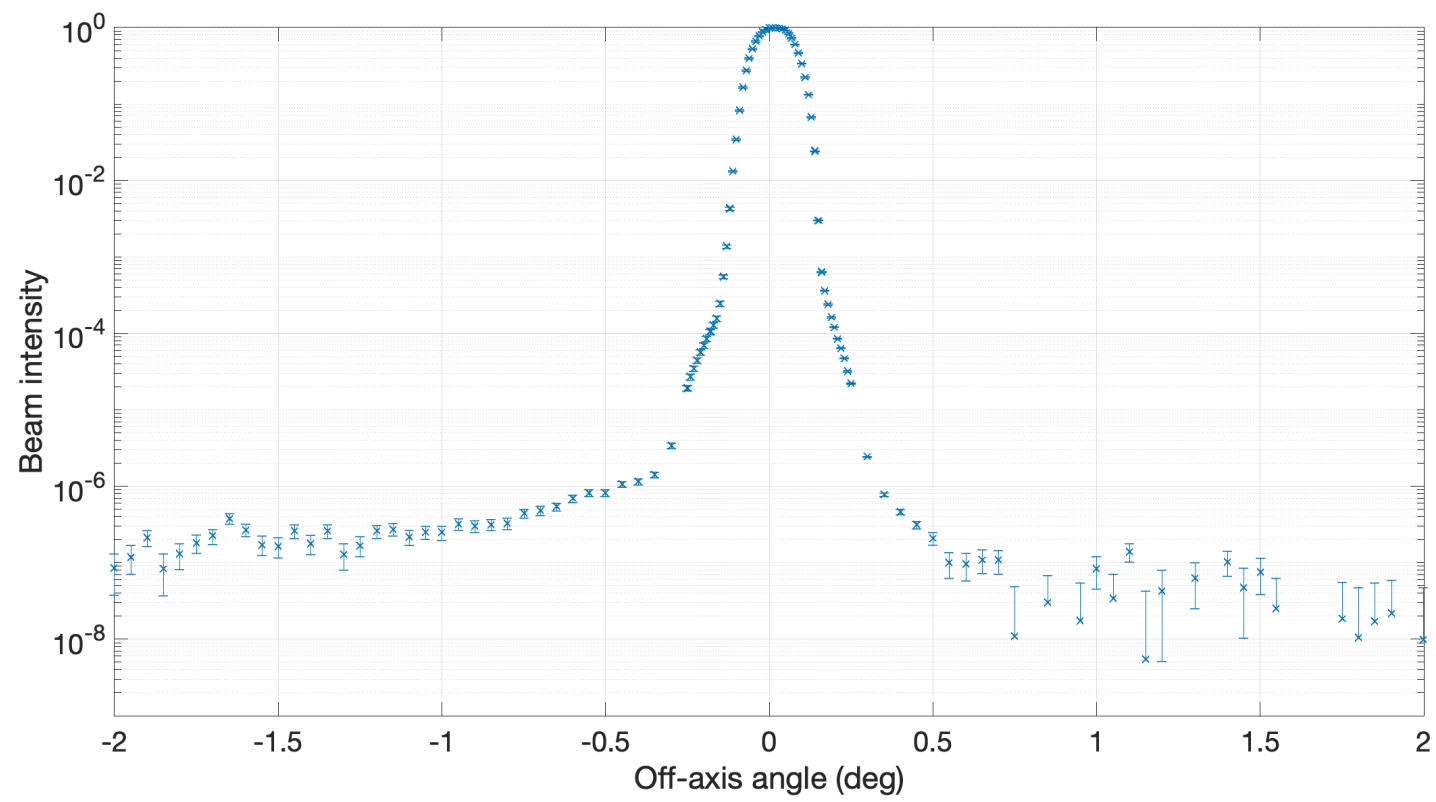

Figure 5: Measurement of the instrumental scatter function using a pre-sample slit width of $0.7 \mathrm{~mm}$ to ensure encompassing the whole beam (collimated to $0.51 \mathrm{~mm}$ ).

The KB mirrors are manufactured to produce a beam of $0.51 \mathrm{~mm}$ width with better collimation than can be obtained by the slits so pre-sample slits are only used to cut away any instrument scatter and down-shaping the beam if needed. Closely scanning the direct beam with a narrow detector slit width of $0.1 \mathrm{~mm}$, the monochromatized beam has FWHM $=0.115 \mathrm{deg} \Leftrightarrow 1.014 \mathrm{~mm}$ measured at $2 \theta$ in the detector. This corresponds to the beam having a divergence of 0.746 arcmin which is in agreement with the expected value from analytical calculations, ${ }^{7}$ confirming proper alignment of the KB mirrors. 


\subsection{Measurement uncertainty and repeatability}

The processing of LEXR data includes evaluation of the statistical uncertainty of each data point and appropriate error propagation through background subtraction and data normalization. Systematic uncertainties and measurement errors are, however, difficult to quantify as they are highly dependent on beamline operator precision in sample mounting, alignment, and general operation of the reflectometer. Sample alignment precision is around $50 \mathrm{\mu m}$ in position and $5 \mathrm{mdeg}$ in angle. Systematic effects such as non-perfect alignment of rotation axes contribute $\sim 3-5$ mdeg. Motor backlash has been measured to shift alignment around 0.3 mdeg. As part of a repeatability study, a sample has been measured 10 times successively and the data is overplotted in Figure 6 .
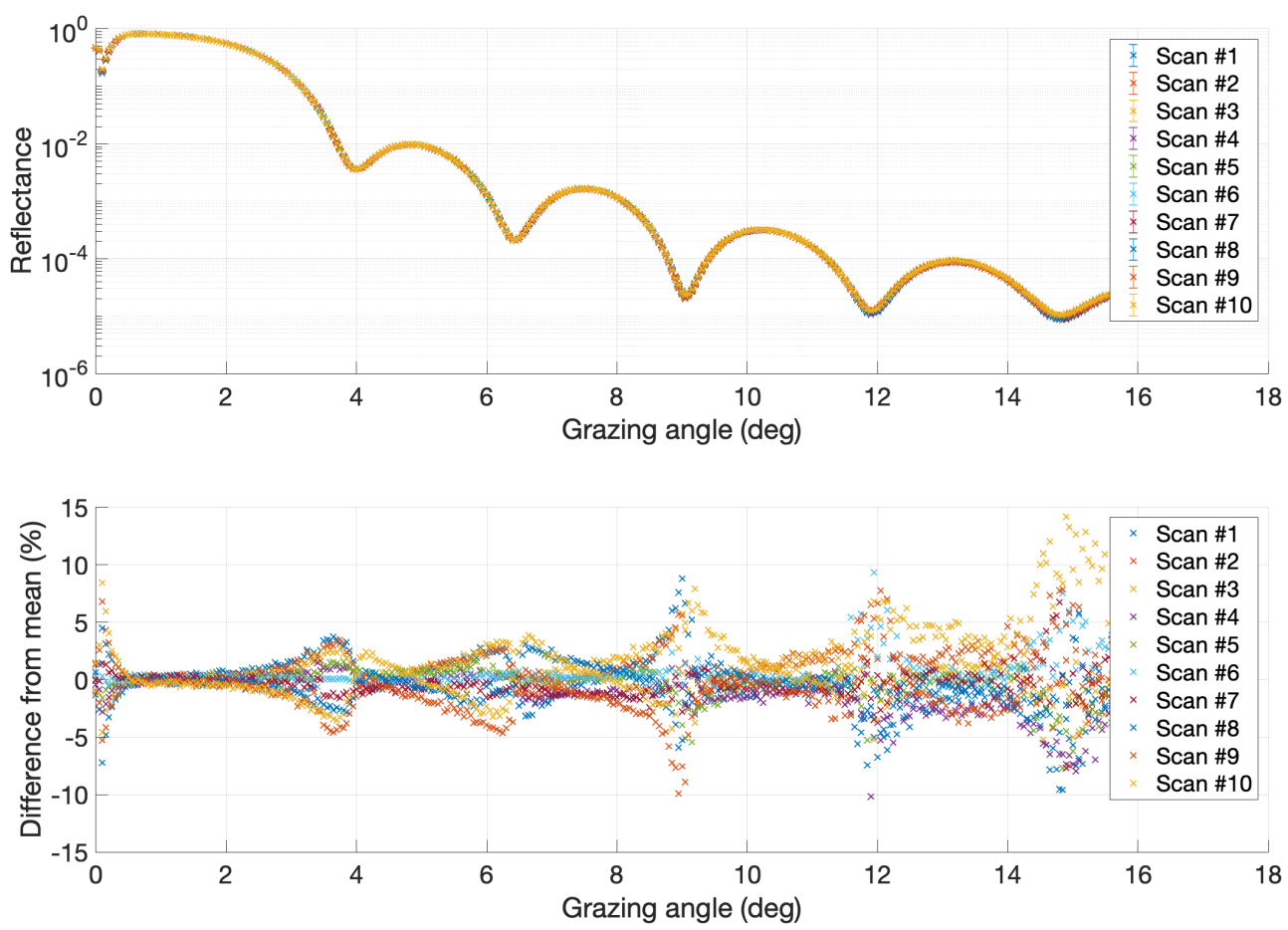

Figure 6: Top: 10 repeated $\theta-2 \theta$ measurements of the same Ir single layer sample. Bottom: Variation in percent from mean value of reflectance at each incidence angle. The data is in statistical agreement but error bars have been omitted here for clarity.

The spread of measured reflectance curves follows a pattern that is inversely correlated with the oscillations of the Kiessig fringes, indicating that a large contributor to the variation in measured reflectance is statistical or signal dependent rather than inconsistency in alignment or motor control. Furthermore the spread in the difference from the mean is centered around 0, meaning that no single measurement deviates substantially from the other nine. There is an increase in variation at the bottom of Kiessig fringes as the signal/noise decreases and, indeed, the largest discrepancies are found at higher grazing angles when the level of noise dominates the measured data. Furthermore, the sawtooth pattern is a clear indication that the main contributor to the difference from the mean is a shift in angle due to motor backlash rather than a signal strength variation. 
Figure 7 shows repeated measurements of the same sample where the vacuum chamber was vented between each measurement, the sample remounted in a different position on the sample holder, realigned in the new position, and measured with different source and detector settings. The repeated measurements again show very similar reflectivity curves, albeit with a larger variation than for measurements performed repeatedly in the same session. While measurements seem to overlap, it is clear from the difference from the mean of the three measurements that different measurement settings result in different levels of uncertainty. It is also clear that there are systematic uncertainties of $\lesssim 3 \%$ that are unaccounted for (evident from the region of total external reflection and the area around the critical angle) and the slope and sawtooth pattern of the difference curves again show that the main contributor to the difference is an alignment shift. The much larger difference between the measurements compared to those shown in Figure 6 is thus due to systematic errors in mounting and alignment precision whereas the previous repeated measurements only had a small systematic shift due to backlash which is an order of magnitude smaller. The best-fit parameters obtained to these measurements are compared in Figure 8. Observing the best-fit parameters and comparing Figures 6 and 7, it seems that sample mounting and alignment are the main contributors to measurement variations. A small drift from one measurement to the next occurs while remounting and realigning a sample which exemplifies the effect of sample misalignment on the systematic uncertainty of the system.
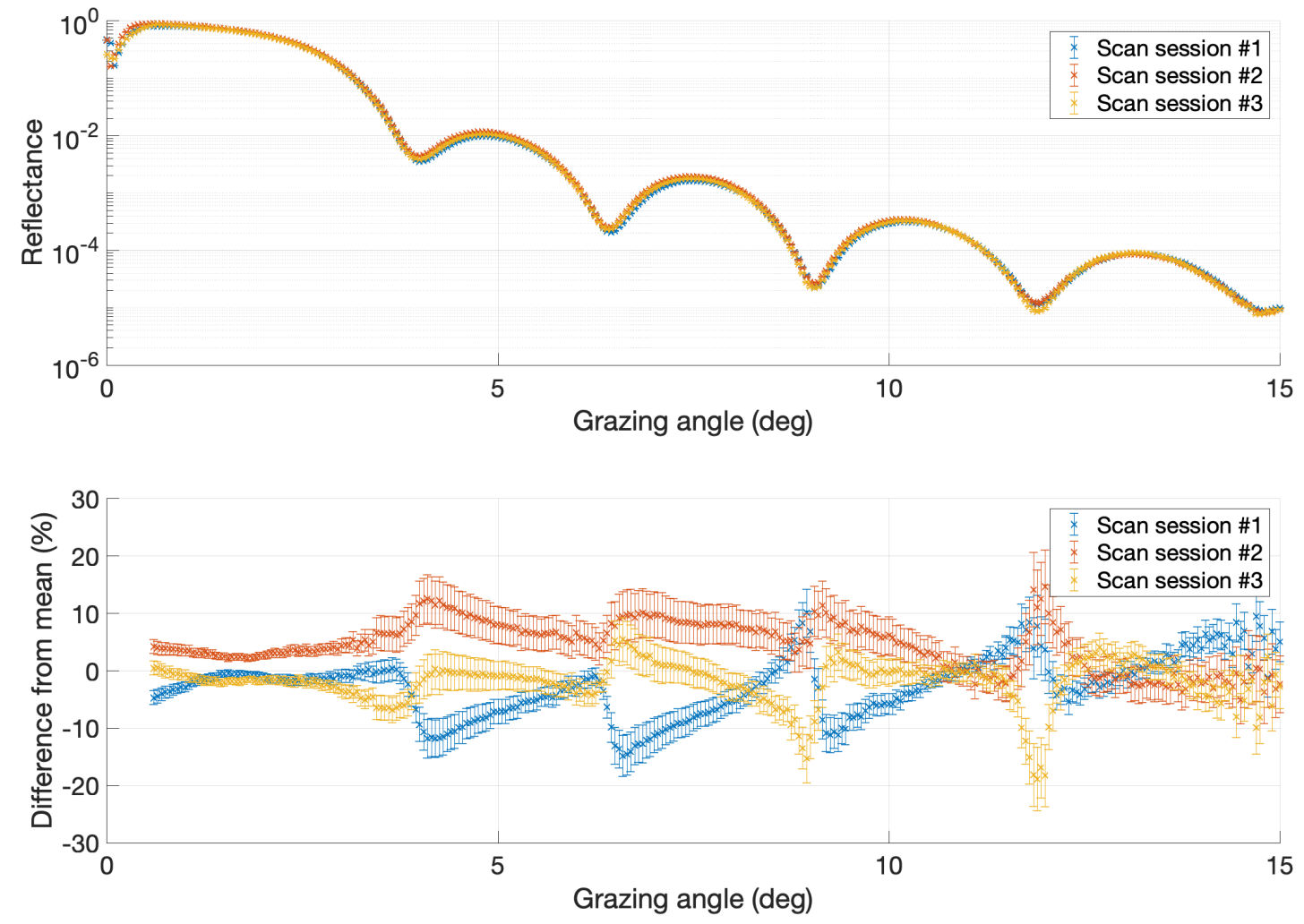

Figure 7: Measurements of the same sample in three different sessions, meaning that the vacuum chamber was vented, the sample remounted in a different position on the sample holder, realigned and remeasured. The difference from the mean below the angle at which the full beam footprint is on the sample has been omitted. 


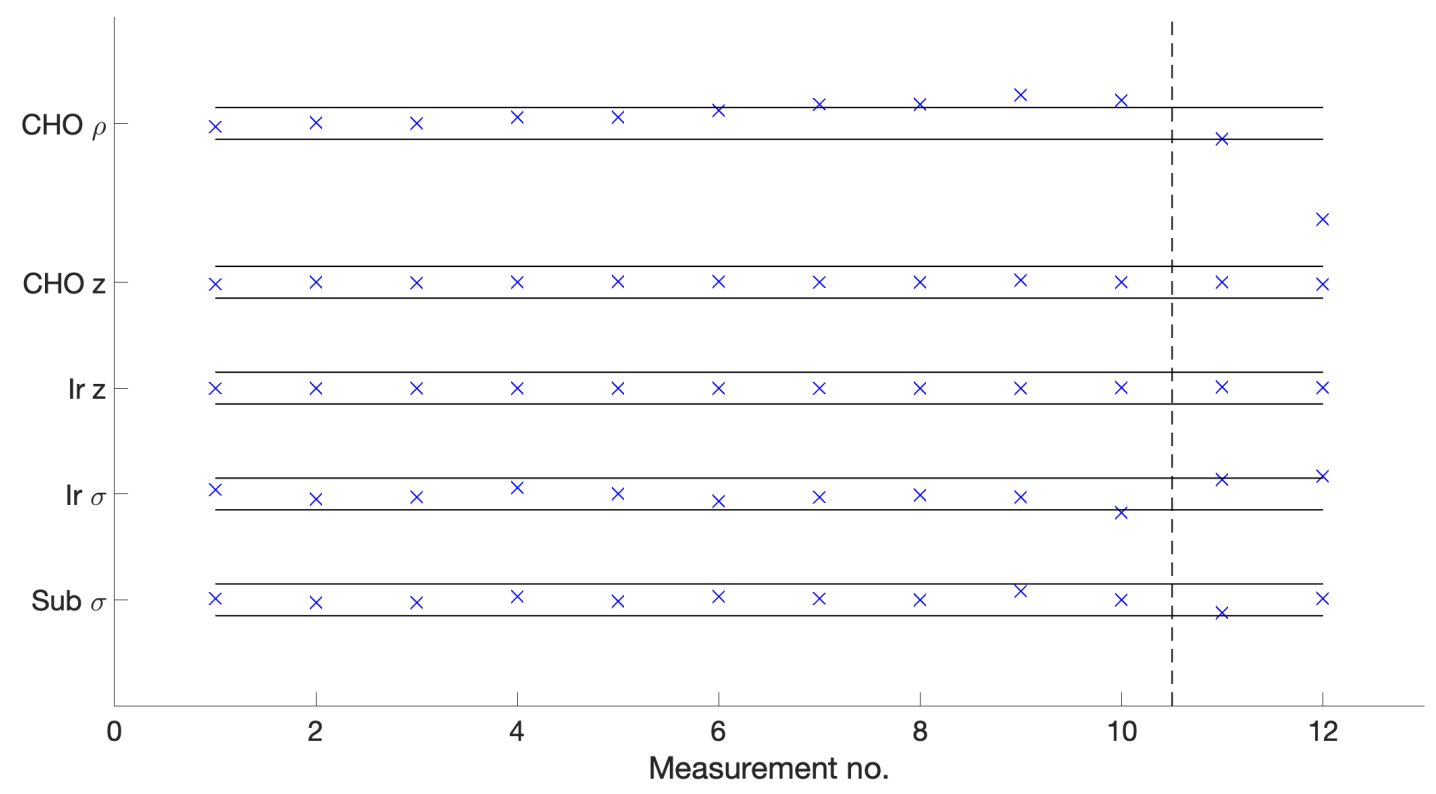

Figure 8: Comparison of best-fit parameters from the repeated measurements of the same sample. Measurements 1-10 were made in succession. Measurements 11 and 12 were each made after remounting and realigning the sample. All points were normalized to the mean obtained value. The black lines indicate $\pm 3 \%$ of the mean fit parameter. $\mathrm{CHO}$ indicates hydrocarbons present on the surface. This could be any C-H-O compound or other contaminants but as this energy is not near any absorption edges, the chemical composition folds into the density parameter.

While the thicknesses of the Ir coating and hydrocarbon overlayer are almost identical in all fits, the roughness parameters vary slightly but largely remain within $\pm 3 \%$. Some systematic error can be seen from the fitted density of the hydrocarbon overlayer. The best fits show a trend of slightly increasing density for each measurement in the same session. This is due to the high sensitivity of this parameter to the critical angle so a slight shift in motor positions due to e.g. backlash when resetting for the next measurement will shift the alignment angle of the sample in the same direction by a small amount. Interpolation from the data shows a maximum difference on angular alignment of 3.7 mdeg with a mean difference between each repeated measurement of 1.2 mdeg. The two measurements that were done after remounting and manually realigning the sample have nearly the same fitted parameters except for the hydrocarbon density which is off by $5 \%$ and $20 \%$, corresponding to roughly 0.1 and $0.3 \mathrm{~g} / \mathrm{cm}^{3}$ respectively, which is likely caused by sample alignment precision. This highlights how sensitive fitting parameters, such as density that relies heavily on the critical angle, are to sample alignment. Other parameters such as coating thickness and roughness have absolute variation in fit parameters of less than $0.1 \AA$, effectively making these small differences in reflectivity curves negligible. The systematic uncertainty originating from alignment variation can not be quantified as the alignment accuracy obtainable is also sample and user dependent. 


\section{LOW-DENSITY OVERLAYER SAMPLE CHARACTERIZATION}

A more natural domain to work in when considering geometric effects is the reciprocal (Fourier) space, in which realspace sample features give rise to a wavelength-independent intensity distribution. For a beam with wavevector $\vec{k}$ reflecting elastically off a sample with angle $\theta$, it can be seen (Figure 9 ) that the momentum transfer vector is $q_{\|}=4 \pi \sin (\theta) / \lambda$. For single layers of low-density materials or uncoated substrates, the limited dynamic range of our $8.048 \mathrm{keV}$ system means that LEXR can probe a larger part of reciprocal space.
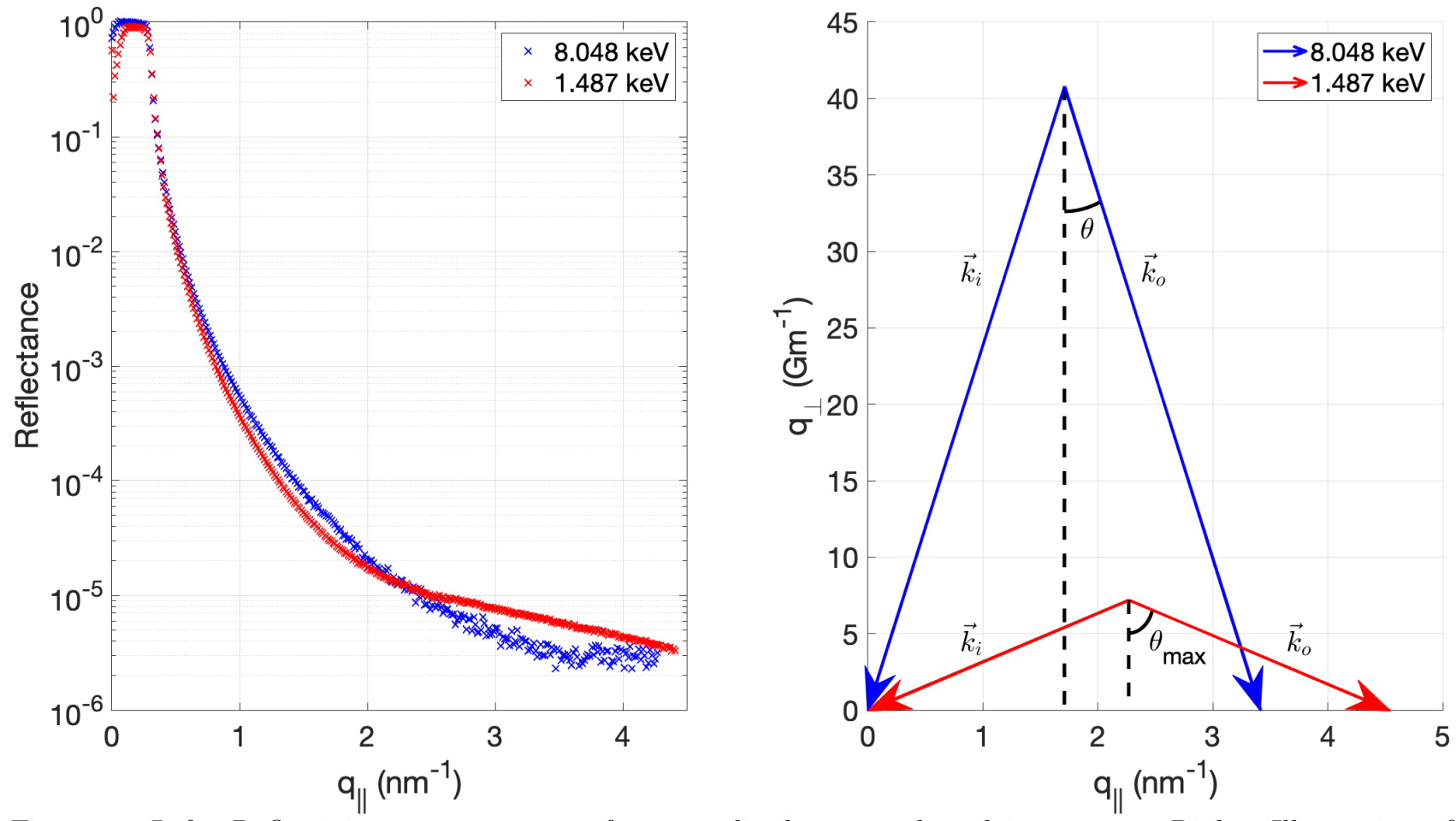

Figure 9: Left: Reflectivity measurements of uncoated substrates plotted in q-space. Right: Illustration of momentum transfer vectors for the two measurements. The $8.048 \mathrm{keV}$ measurement is limited to $q_{\|} \approx 3.4 \mathrm{~nm}^{-1} \Leftrightarrow 2.4 \mathrm{deg}$ by the dynamic range, whereas the $1.487 \mathrm{keV}$ measurement is limited by chamber geometry to $\theta_{\max }=17.5 \operatorname{deg} \Leftrightarrow 4.53 \mathrm{~nm}^{-1}$.

The curves in Figure 9 (left) do not overlap entirely due to a hydrocarbon overlayer modifying the low-energy reflectance.

Simulations show (Figure 10) that a smooth hydrocarbon overlayer on $10 \mathrm{~nm}$ Ir will give rise to interference structures in the region $q_{\|} \sim 8-10 \mathrm{~nm}^{-1}, 7-8$ orders of magnitude down, corresponding to 5.6-7 deg at $8.048 \mathrm{keV}$ or 32-42 deg at $1.487 \mathrm{keV}$. This is beyond the mechanical range of LEXR and beyond the dynamic range of our available $8.048 \mathrm{keV}$ reflectometer. This effect should, however, be obtainable from synchrotron measurements. 


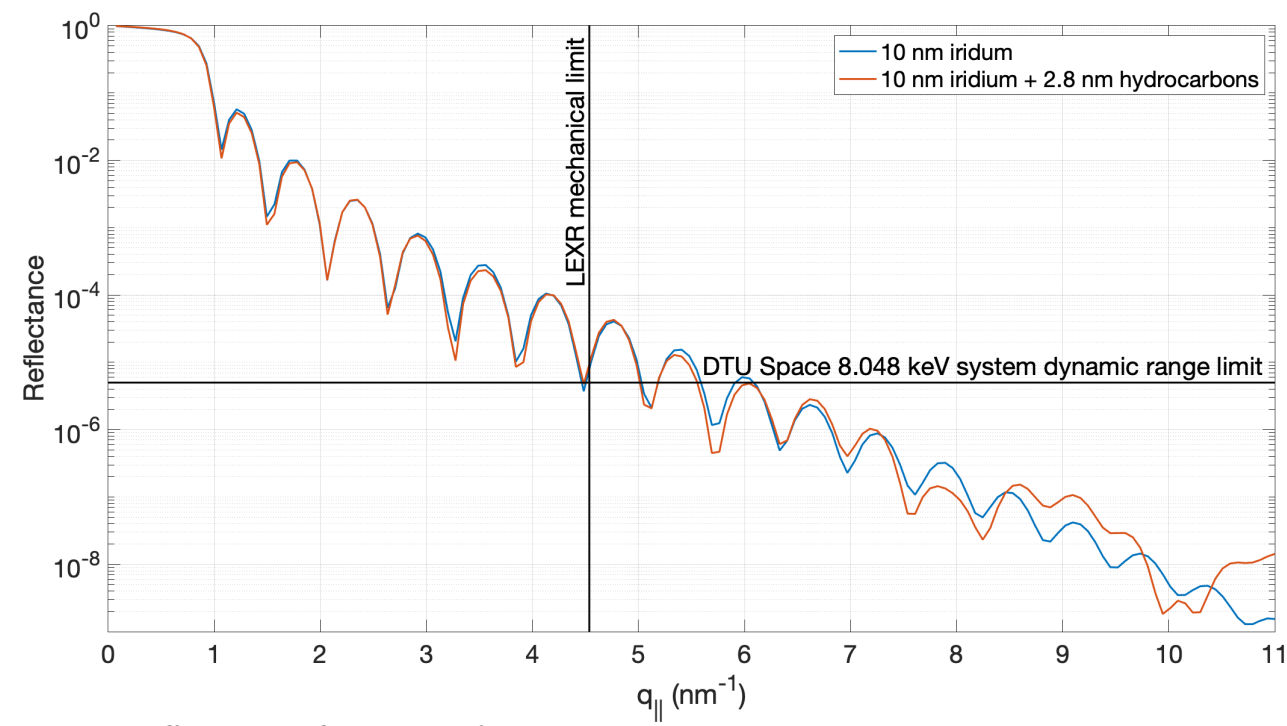

Figure 10: Simulated effect on reflectance of a smooth hydrocarbon overlayer on $10 \mathrm{~nm}$ Ir plotted in reciprocal space. Note from the limits of the two reflectometers that the interference region $8-10 \mathrm{~nm}^{-1}$ can not be reached by either system.

The dynamic range of the $8.048 \mathrm{keV}$ system at DTU Space is $5 \times 10^{-6}$ and the vacuum chamber geometry of LEXR effectively limits its reciprocal spatial range to $4.5 \mathrm{~nm}^{-1}$. Other than this purely geometric consideration, low-density overcoats such as naturally occurring hydrocarbons do, however, also alter the shallow-angle reflectance due to absorption of low-energy X-rays, an effect not present at higher energies. This effect means that hydrocarbons can be modelled and must be accounted for in LEXR measurements as shown in Figure 11.

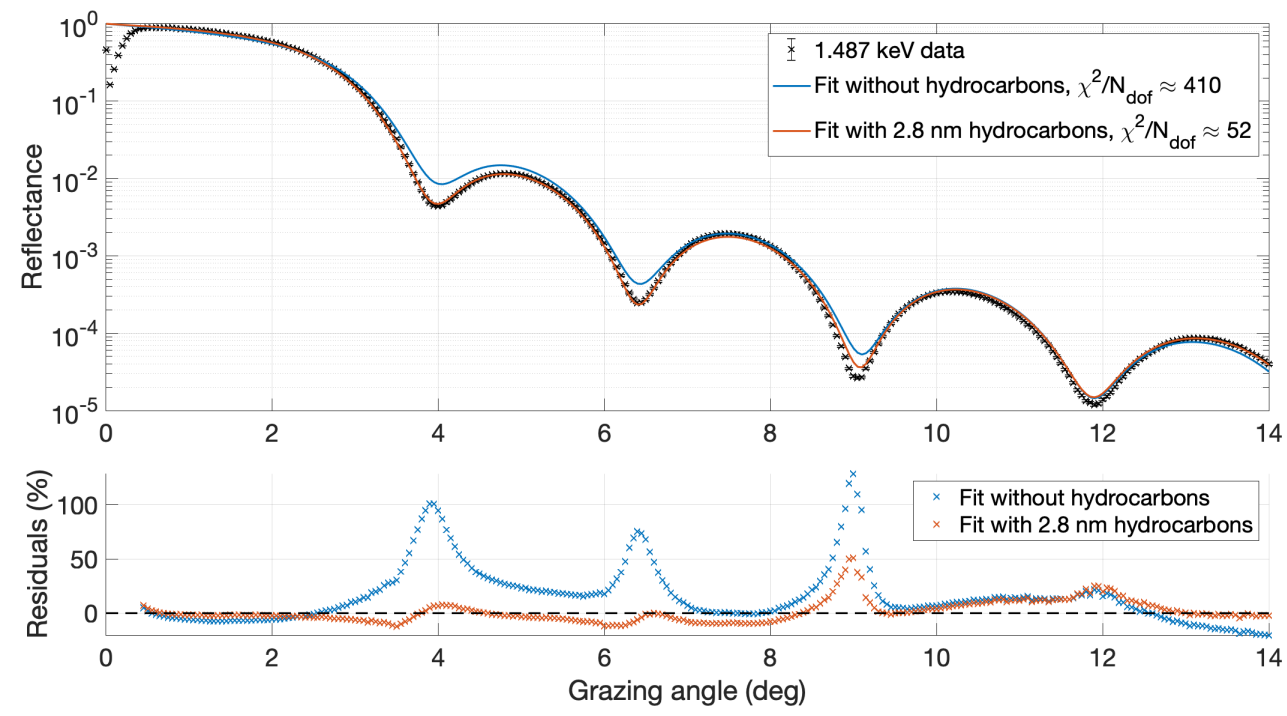

Figure 11: Comparison of fits to a single layer Ir coated sample using models with and without hydrocarbon overlayers.

Due to the measurement errors only accounting for statistical and not systematic uncertainties, the errors are underestimated so even for the better fit, the reduced $\chi^{2}$ is rather large. The method can nevertheless be used to compare several models and determine which one is better for the sample in question. Adding an uncertainty of around $3 \%$ of the signal as discussed in relation to Figure 7, the reduced $\chi^{2}$ becomes 6 . 


\section{INSTRUMENT CHARACTERISTICS}

\subsection{Small-beam intensity variation}

The beam cross section is rather non-uniform with the largest pixel-to-pixel variations being around $30 \%$ near the sides (see Figure 12) and 10-15\% near the center of the beam.
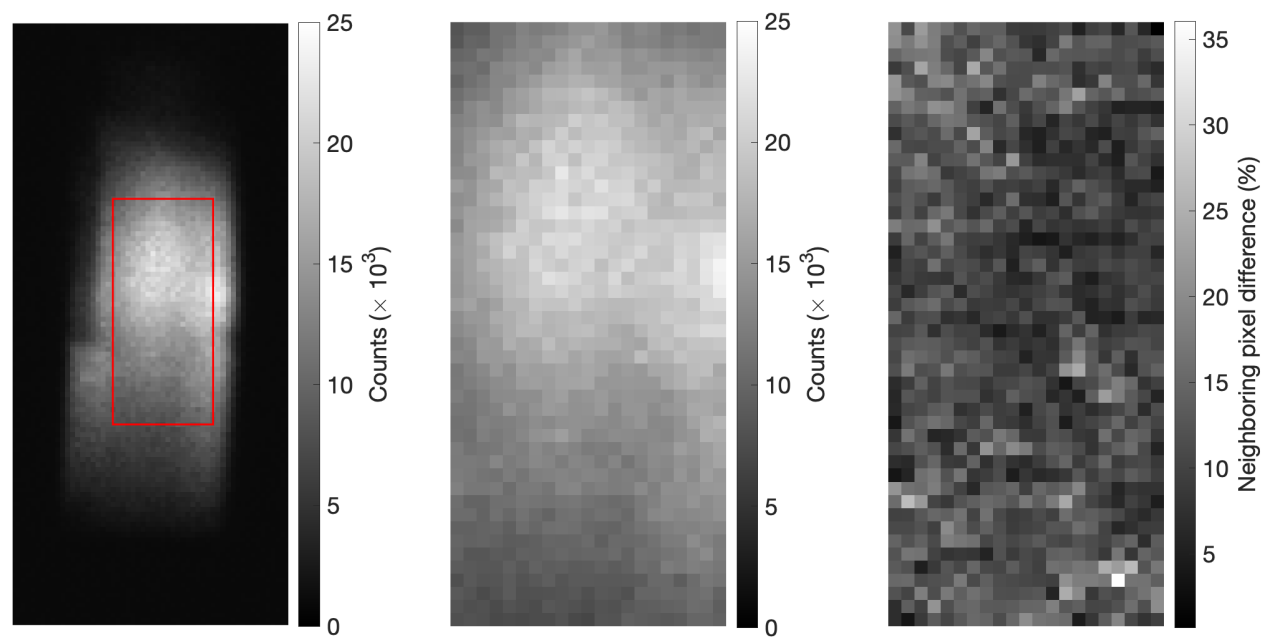

Figure 12: Left: Full beam as seen from the detector. Mid: Subsection of beam, corresponding to red box on left image, ensuring the beam edge is excluded. Right: Largest signal strength difference to any of the 8 neighboring pixels within the beam subsection.

This is irrelevant for measurements where a large portion of the beam is used and the full beam footprint is on the sample. For pencil-beam measurements, however, the slits are reduced so as to only choose a small subsection of the beam cross section and variation in the integrated flux therefore induces large uncertainty in the measured data. Figure 13 shows the direct beam intensity as function of time for different slit sizes.

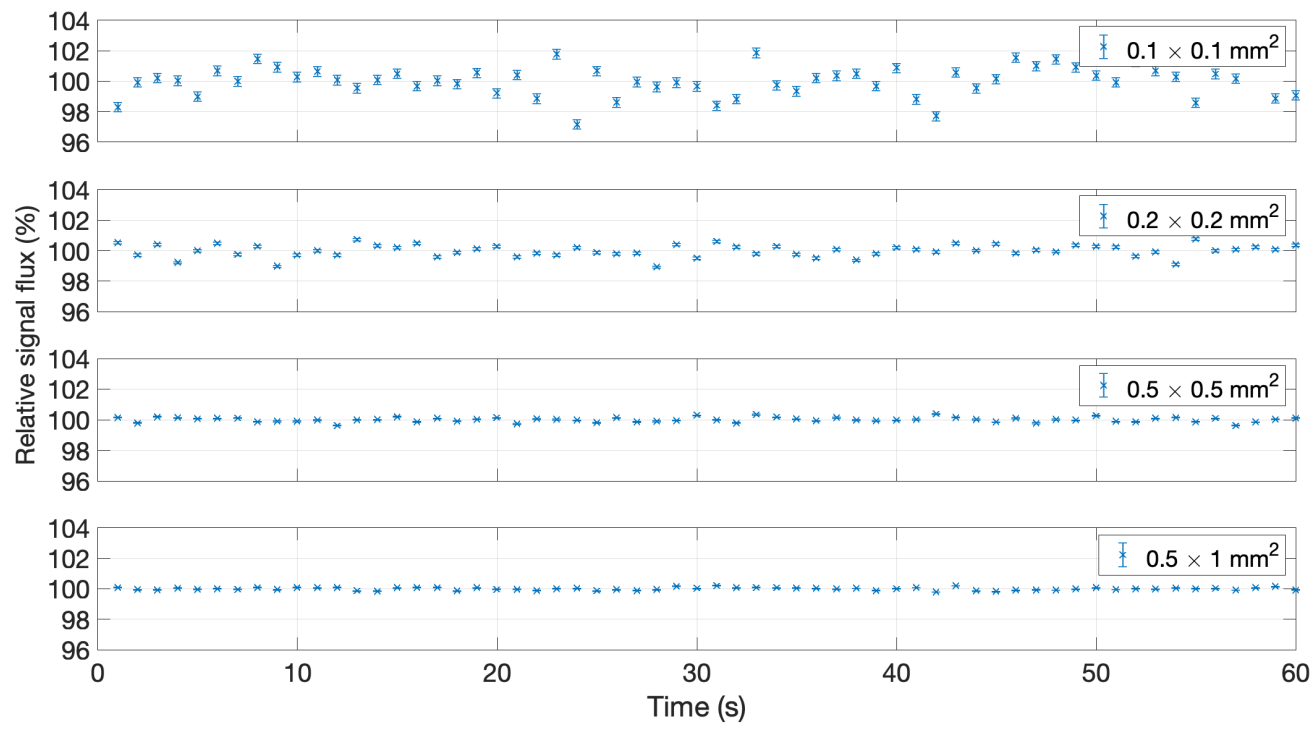

Figure 13: Variation of beam intensity relative to the mean over $60 \mathrm{~s}$ with a $1 \mathrm{~s}$ sampling frequency for different slit sizes. 
Variations of the same magnitude are observed for exposures with open slits but where subsections of pixels are chosen in post-processing to match the size of the slits used to obtain Figure 13. The cause of the variation is thus either constant minor flux variations across the beam cross section, or vibrations induced on the reflectometer from e.g. the turbo pump causing shifts in the flux distribution map. In any case this effect is averaged out so beams larger than $0.2 \mathrm{~mm}^{2}$ have integrated flux variation less than $1 \%$.

\subsection{Bandwidth limit on roughness determination}

Typically, measurements are performed with an ROI spanning 80 pixels in width which, with a pixel pitch of $26 \mathrm{\mu m}$, corresponds to around $2 \mathrm{~mm}$. With a sample-to-detector distance of $505 \mathrm{~mm}$, the maximal scatter acceptance angle is around $\Delta \theta_{s} \approx 7$ arcmin. From the more detailed scatter geometry illustrated in Figure 14, it can be seen that, generally, the width of the scatter distribution follows

$$
\Delta \theta \approx \frac{2 \lambda}{\xi \sin \left(\theta_{i}\right)}
$$

As the incidence angle $\theta_{i}$ decreases, the scatter angle eventually becomes parallel with the mirror surface, after which the width of the distribution becomes independent of incidence angle $\Delta \theta \approx \sqrt{2 \lambda / \xi}$.

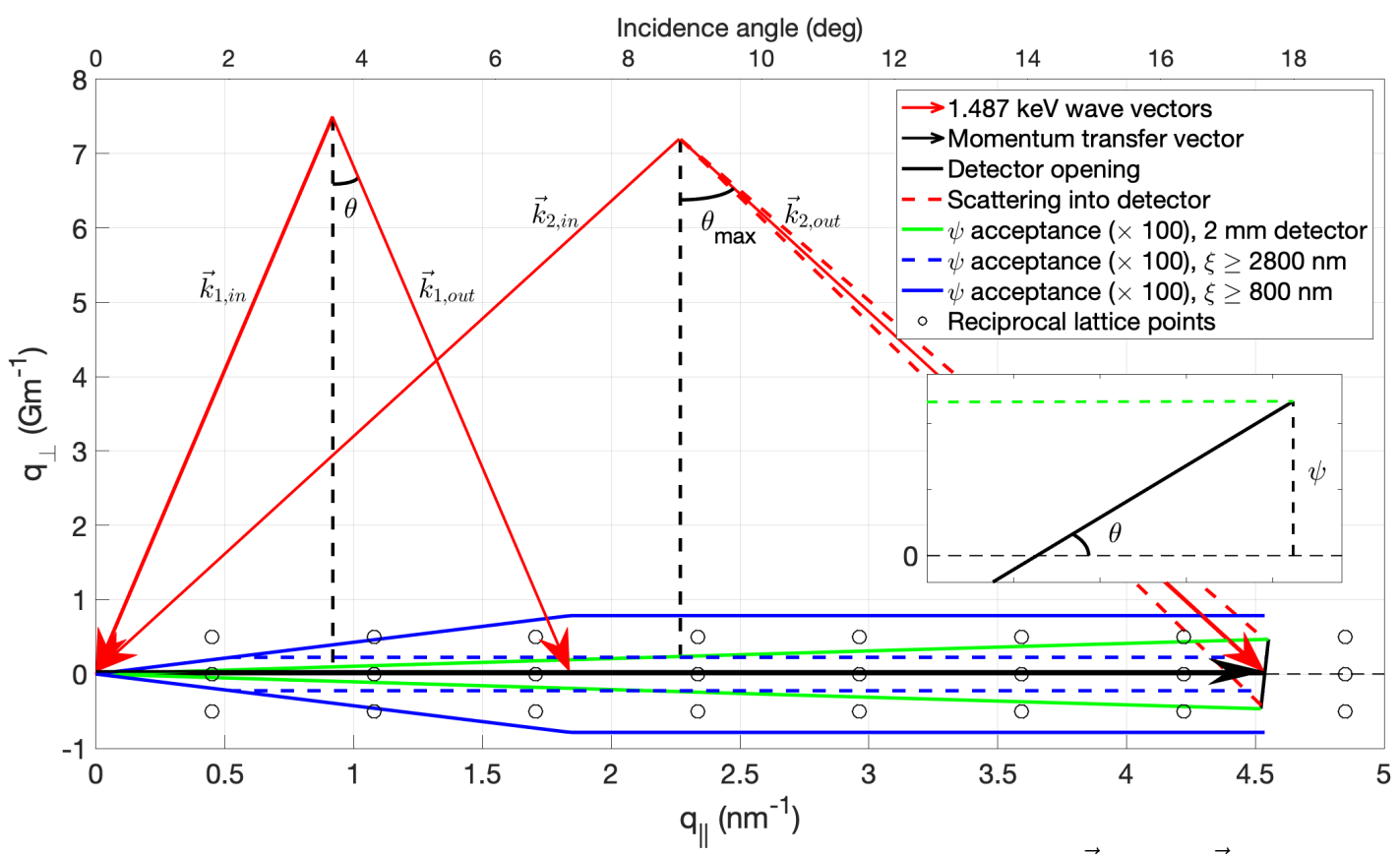

Figure 14: Illustration of the LEXR scattering geometry in reciprocal space. $\vec{k}_{\text {in }}$ and $\vec{k}_{\text {out }}$ are the incoming and outgoing wave vectors. $\theta$ is the beam incidence angle on the sample and $\psi=2 \pi / \xi$ is the reciprocal transverse characteristic length. The $\psi$ acceptance region is indicated for a detector of fixed width throughout the measurement as well as where the detector acceptance width is varied to probe a constant range of correlation lengths. The angles have been greatly exaggerated for illustration purposes. The spacing of reciprocal lattice points along $q_{\|}$are those of a $10 \mathrm{~nm}$ coating, their spacing in $q_{\perp}$ is arbitrary. The insert shows the geometry of the transverse probing length at any point during the measurement. 
For LEXR with $\lambda \approx 8.34 \AA, \Delta \theta_{s} \approx 7 \operatorname{arcmin}$, and $\theta_{i}=5$ deg we have $\xi \approx \lambda /\left(\Delta \theta_{s} \theta_{i}\right) \approx 4.7 \mu$ m meaning that fitting to the specular measurement around 5 degrees will give information about the roughness with $\xi<4.7 \mu \mathrm{m}$ and, more generally, information can only be extracted about features with correlation lengths no larger than a few tenths of microns. Alternatively, the detector acceptance angle can be varied during the scan such that the probed correlation lengths remain constant. Investigating, for example, the roughness region with $2800 \mathrm{~nm} \geq \xi \geq 800 \mathrm{~nm}$, one could compare reflectivity measurements where the detector opening varies from fully open down to $0.8 \mathrm{~mm}$ and $2.8 \mathrm{~mm}$ at $17.5 \mathrm{deg}$ respectively. The detector slit is placed $410 \mathrm{~mm}$ from the sample and its maximal width of $7 \mathrm{~mm}$ limits the accepted total scatter width to $\Delta \theta_{\max } \approx 58.7$ arcmin which in turn limits the characteristic length to $\xi \sin \left(\theta_{i}\right) \gtrsim 97.6 \mathrm{~nm}$.

Carrying out such a measurement on a sample with a $10 \mathrm{~nm}$ Ir coating on a NuSTAR-type glass substrate that also includes a "standard scan" of constant $2 \mathrm{~mm}$ acceptance width on the detector, we find that the measured reflectance falls slightly below the others on the standard scan up to around 5 deg (shown in Figure 15). From around 7-8 deg the measured intensity on the $2800 \mathrm{~nm}$ scan falls below the other scans which is also where the two $\psi$ acceptance curves intersect as indicated on Figure 14. From this point on there is a clear, albeit small $(\sim 8 \%)$, difference such that $\mathrm{R}(\xi \geq 800 \mathrm{~nm})>\mathrm{R}(2 \mathrm{~mm}$ acceptance $)>\mathrm{R}(\xi \geq 2800 \mathrm{~nm})$ until around $\theta_{i} \sim 13.5$ deg from which point on $\mathrm{R}(\xi \geq 800 \mathrm{~nm}) \approx \mathrm{R}(2 \mathrm{~mm}$ acceptance $)>\mathrm{R}(\xi \geq 2800 \mathrm{~nm})$, indicating that the sample has at least one roughness correlation length $\xi \approx 1.7 \mu \mathrm{m}$.

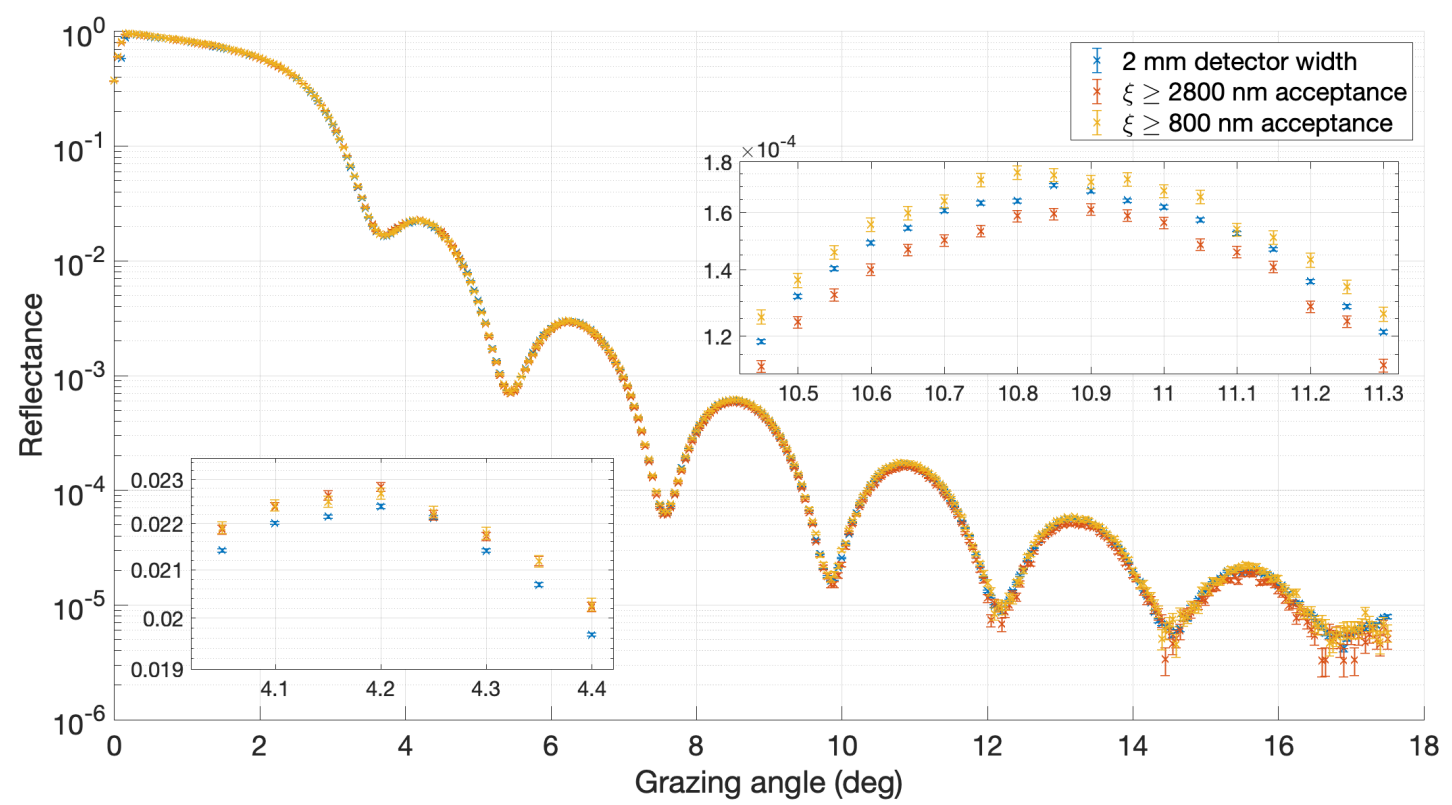

Figure 15: Repeated measurements of a NuSTAR-type glass substrate coated with $10 \mathrm{~nm}$ Ir with different acceptance widths at the detector. Inserts show close-ups of two parts of the scan.

Performing the same type of measurements on a flat Si wafer coated with $10 \mathrm{~nm}$ Ir, the three measurements agree to within the statistical uncertainty of the measurements, indicating an exclusion region $\xi<800 \mathrm{~nm} \vee \xi>2800 \mathrm{~nm}$. Furthermore, the agreement of the reflectivity measurements for different ROIs on the detector verifies the background substraction method. 
Measuring the reflectance with the sample and detector moving with the ratio $1: 2$ but with the sample stage slightly offset from zero gives a measurement along $q_{\|}$but offset in $q_{\perp}$. This probes the scattering component close to the specular direction which is assumed to be similar to the amount of scattering hidden within the specular beam. In principle, this should be subtracted from the first measurement to remove the component of scattered light hidden within the specular beam but as shown in Figure 16, this effect is sufficiently small to be negligible in data fitting. The scattering from Kiessig fringes gives rise to streaks out to $2 \pi / \xi$ in $q_{\perp}$ and these are clearly recorded on the offset measurement of the coated glass substrate. This measurement is offset by about the same angle as the detector acceptance width at high angles and the $\xi \geq 800 \mathrm{~nm}$ scan has a width about twice as large as the standard scan. We see that, indeed, the difference between these two scans on Figure 15 is the same order of magnitude as the amount of scattering measured at the offset. The offset specular measurements also agree with the equivalence of the three types of measurements on the coated $\mathrm{Si}$ wafer as the offset measurement here indicates only very large-scale roughness correlation on the order of several tens of micrometers.

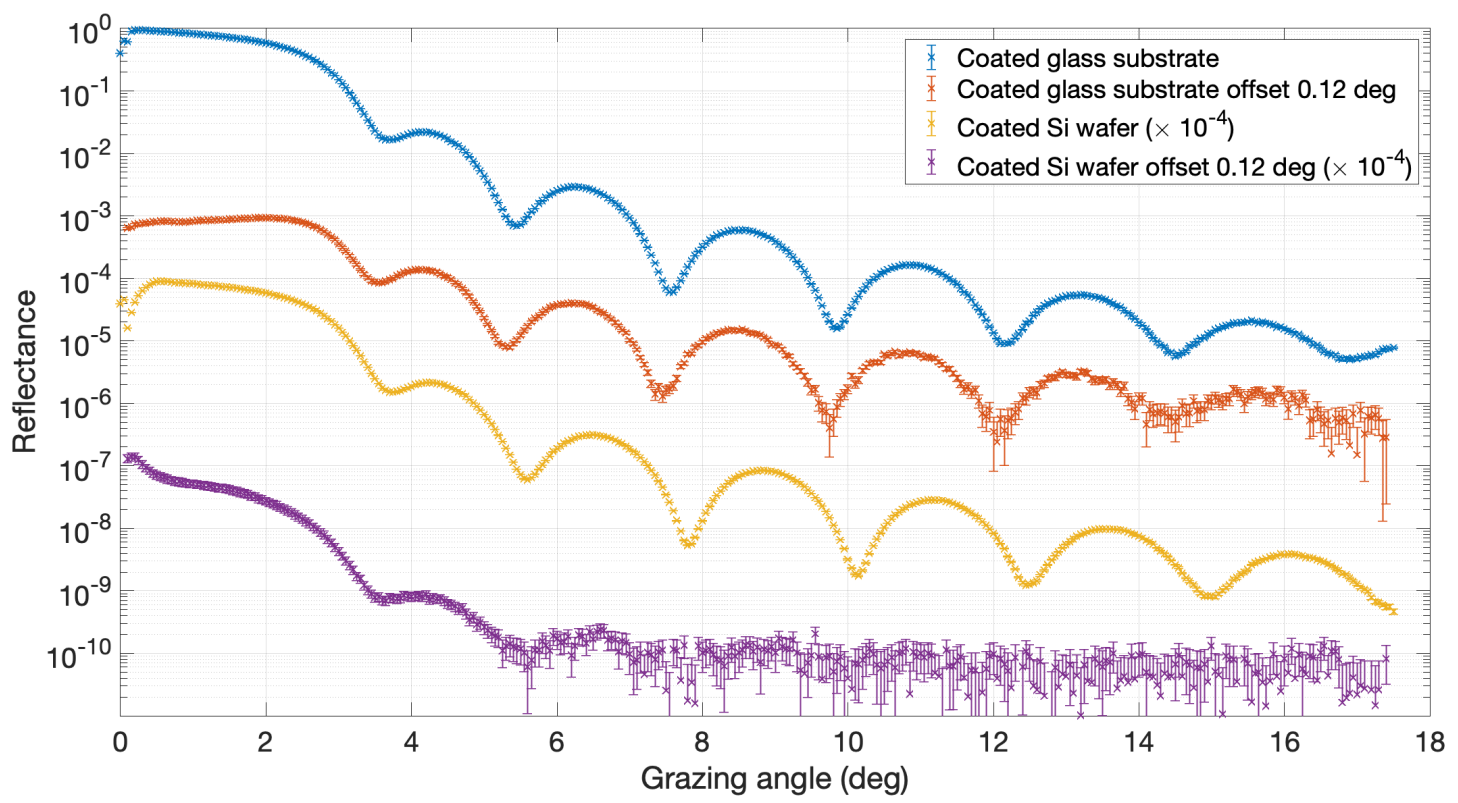

Figure 16: Specular and offset specular measurements on NuSTAR-type glass substrate and flat Si wafer coated with $\sim 10 \mathrm{~nm}$ Ir.

By inserting the Bragg equation into eq. (1), one can see that $\Delta \theta \propto d / \xi$ independent of wavelength. Bragg features occur at larger angles for lower energies so a larger $\Delta \theta$ is available before shadowing effects from the sample appear, meaning that for non-specular scatter measurements, low-energy reflectometers can give information about smaller $\xi$.

\section{SUMMARY}

The performance of each of the components, as well as the LEXR system as a whole, has been evaluated. The reflectometer has a dynamic range of 6-8 orders of magnitude depending on measurement conditions, a beam purity of $99.3 \%$ and a divergence of 0.746 arcmin. We have evaluated how the system can be used to probe different characteristic length scales and discussed the bandwidth limits for different measurement techniques. Lastly, we have demonstrated measurement repeatability and discussed the effects of hydrocarbons and other surface contaminants on the sample reflectance. 


\section{ACKNOWLEDGEMENTS}

This activity has been funded by the European Space Agency under contract 4000102248/10/NL/PM.

\section{REFERENCES}

[1] K. Nandra, D. Barret, X. Barcons, et al. The Hot and Energetic Universe: A White Paper presenting the science theme motivating the Athena+ mission. astro-ph.HE, arXiv:1306.2307, 2013.

[2] A. Abeln et al. Conceptual Design of BabyIAXO, the intermediate stage towards the International Axion Observatory, 2020.

[3] Jessica A. Gaskin et al. Lynx X-Ray Observatory: an overview. Journal of Astronomical Telescopes, Instruments, and Systems, 5(2):1 - 15, 2019.

[4] D. D. M. Ferreira, S. Massahi, F. E. Christensen, et al. Design, development, and performance of X-ray mirror coatings for the ATHENA mission. Proceedings of SPIE, 10399, 2017.

[5] S. Massahi, L. M. Vu, D. D. M. Ferreira, et al. Balancing of residual stress in thin film iridium by utilizing chromium as an underlayer. Proceedings of SPIE, 11444, 2020.

[6] S. Svendsen, D. D. M. Ferreira, S. Massahi, et al. Status of the Ir/SiC coating development for the Athena optics. Proceedings of SPIE, 11444, 2020.

[7] P. L. Henriksen, F. E. Christensen, S. Massahi, D. D. M. Ferreira, S. Svendsen, A. Jafari, and B. Shortt. LEXR: A low-energy X-ray reflectometer for characterization of ATHENA mirror coatings. Proceedings of SPIE, 11119, 2019.

[8] William W. Craig et al. Fabrication of the NuSTAR flight optics. Proceedings of SPIE, 8147, 2011. 